\title{
hsa-miR-15b-5p regulates the proliferation and apoptosis of human vascular smooth muscle cells by targeting the ACSS2/PTGS2 axis
}

\author{
SHUJIE GAN ${ }^{1}$, JIEQI MAO ${ }^{1}$, YUQIN PAN ${ }^{2}$, JINGDONG TANG $^{3}$ and ZHENGJUN QIU ${ }^{1}$ \\ Departments of ${ }^{1}$ Vascular Surgery and ${ }^{2}$ Nursing, Shanghai General Hospital, \\ Shanghai Jiao Tong University School of Medicine, Shanghai 200080; ${ }^{3}$ Department of Vascular Surgery, \\ Shanghai Pudong Hospital Affiliated to Fudan University, Shanghai 201399, P.R. China
}

Received October 16, 2020; Accepted May 12, 2021

DOI: $10.3892 /$ etm.2021.10642

\begin{abstract}
A previous bioinformatic analysis from our group predicted that the interaction of microRNA (miRNA/miR)-15b with the acyl-CoA synthetase short chain family member 2 (ACSS2) gene was important for the development of abdominal aortic aneurysm (AAA). Apoptosis of aortic vascular smooth muscle cells (VSMCs) is a pathological feature of AAA. The present study aimed to explain the roles of miR-15b/ACSS2 in AAA by exploring their effects on the proliferation and apoptosis of aortic VSMCs. Human aortic VSMCs (T/G HA-VSMC cell line) were divided into six groups and were transfected with miR-15b-5p mimics, mimic negative control (NC), miR-15b-5p inhibitors, inhibitor NC, miR-15b-5p mimics+pcDNA3.1 and miR-15b-5p mimics+ACSS2-overexpessing vector. CCK-8 assay was used to determine cell proliferation. Annexin V-FITC/PI staining and flow cytometry assays were used to measure cell apoptosis. Dual-luciferase reporter assays were used to confirm the targeted relationship between miR-15b-5p and ACSS2. Reverse transcription-quantitative PCR and/or western blotting were used to examine the expression levels of miR-15b-5p, ACSS2 and prostaglandin-endoperoxide synthase 2 (PTGS2). Following transfection of T/G HA-VSMCs with mimics and inhibitors to respectively upregulate and downregulate miR-15b-5p, the results demonstrated that overexpression of miR-15b-5p inhibited cell proliferation and
\end{abstract}

Correspondence to: Dr Zhengjun Qiu, Department of Vascular Surgery, Shanghai General Hospital, Shanghai Jiao Tong University School of Medicine, 100 Haining Road, Shanghai 200080, P.R. China E-mail: qiuzhengjun2020@163.com

Dr Jingdong Tang, Department of Vascular Surgery, Shanghai Pudong Hospital Affiliated to Fudan University, 2800 Gongwei Road, Shanghai 201399, P.R. China

E-mail: drtangjingdong@126.com

Key words: abdominal aortic aneurysm, aortic vascular smooth muscle cells, apoptosis, proliferation, microRNA-15b-5p, acyl-CoA synthetase short chain family member 2 promoted cell apoptosis; silencing of miR-15b-5p obtained the opposite results. ACSS2 may be a direct target of miR-15b-5p, since the luciferase activity of a ACSS2 wild-type vector, but not that of a ACSS2 mutant reporter, was significantly inhibited by miR-15b-5p mimics compared with controls. Additionally, the expression levels of ACSS2 and its downstream gene PTGS2 were significantly reduced or increased following transfection with miR-15b-5p mimics or inhibitors, respectively. Furthermore, overexpression of ACSS2 reversed the antiproliferative and proapoptotic effects of miR-15b-5p mimics by blocking the production of PTGS2 protein. In conclusion, miR-15b-5p may promote the apoptosis and inhibit the proliferation of aortic VSMCs via targeting the ACSS2/PTGS2 axis. The present study provided preliminary evidence indicating that the miR-15b-5p/ACSS2/PTGS2 axis may be a potential target for the treatment of AAA.

\section{Introduction}

Abdominal aortic aneurysm (AAA), defined as a focal dilation of the abdominal aorta by $\geq 1.5$ times the normal diameter or to a diameter of $>3 \mathrm{~cm}$ (1), is a common, progressive and lethal vascular disease in adults aged $>65$ years (2). It was estimated that there were 2,347,339 cases and 41,374 deaths attributable to AAA in 2013 in the USA (3). The incidence of AAA was 27.9 per 100,000 in men and 3.3 per 100,000 in women, and the mortality due to rupture was $59.2 \%$ in men and $48.8 \%$ in women in Germany in 2014 (4). Currently, surgical repair (including open and endovascular) is the mainstay treatment for AAA, but long-term prognosis remains unsatisfactory, with an 8-year survival rate of $50 \%$ (5). Therefore, it is essential to develop novel therapeutic options.

Although the pathogenesis is complex, loss of aortic vascular smooth muscle cells (VSMCs) is an evident pathological feature of AAA (6). Accumulating studies have found that microRNAs (miRNAs/miRs), small non-coding RNA molecules comprised of 18-25 nucleotides, are aberrantly expressed in AAA and could control the proliferation and apoptosis of aortic VSMCs by binding to the 3'-untranslated region (3'-UTR) of target mRNAs and then inhibiting their expression post-transcriptionally. Liang et al (7) detected 
that the expression of miR-195 was significantly increased in the aortic media of patients with AAA compared with normal non-aneurysmal tissues. Overexpression of miR-195 in human aortic VSMCs led to a significant decrease in cell proliferation and an increase in cell apoptosis, while transfection with anti-miR-195 obtained the opposite results. Luciferase reporter assays and rescue studies indicated that SMAD family member 3 (Smad3) may be a downstream target of miR-195 (7). Zhao et al (8) identified that the expression levels of miR-7 were upregulated in aortic specimens obtained from patients with AAA compared with aortic specimens from controls. Knockdown of miR-7 increased the proliferation and suppressed the apoptosis of primary VSMCs isolated from aortic specimens of patients with AAA (8). Luciferase reporter, reverse transcription-quantitative PCR (RT-qPCR) and western blot assays suggested that miR-7 may function by directly targeting cytoskeleton associated protein 4 (8). Cao et al (9) observed that miR-504 was less expressed in the aortic cells derived from patients with AAA compared with that in the controls. Cell growth was significantly increased in VSMCs transfected with miR-504 vectors compared with those with non-targeting controls (9). The tumor suppressor p53 was the direct target of miR-504 (9). Furthermore, miR-129-5p, miR-155-5p and $\mathrm{miR}-28-5 \mathrm{p}$ were also reported to be apoptosis-related drivers for AAA by targeting Wnt family member 5A (10), Fos proto-oncogene/Zic family member 3 (ZIC3) (11) and glutamate ionotropic receptor AMPA type subunit 4/LY6/PLAUR domain containing 3 (12), respectively. By contrast, miR-21a/miR-26a and miR-126 may exert protective effects on the development of AAA by modulating phosphatase and tensin homolog (PTEN) $(13,14)$ and ADAM metallopeptidase domain 9 (15), respectively. These findings implied that miRNAs may represent important targets for the development of anti-AAA agents.

A previous study from our group integrated the miRNA and mRNA expression profile datasets of aortic samples from patients with AAA and identified several novel miRNA/mRNA interaction pairs that may be crucial in the development of human AAA. These previous results included the predicted interaction pair of miR-15b (which was upregulated in AAA) with the mRNA for acyl-CoA synthetase short chain family member 2 (ACSS2; which was downregulated in AAA) (16). Therefore, the present study aimed to validate the regulatory relationship between miR-15b and ACSS 2 by using a luciferase reporter assay, and to explore their roles in the proliferation and apoptosis of aortic VSMCs by in vitro overexpression or knockdown experiments. The present results may provide preliminary evidence to suggest that miR-15b/ACSS2 may be a potential therapeutic target for AAA.

\section{Materials and methods}

Cell culture. A human aortic VSMC line (T/G HA-VSMC) was purchased from Procell Life Science \& Technology Co., Ltd. Human aortic VSMCs were cultured in smooth muscle cell medium (SMCM; ScienCell Research Laboratories, Inc.) which contained basic medium, $2 \%$ fetal bovine serum, $1 \%$ smooth muscle cell growth supplement and $1 \%$ penicillin/streptomycin solution. A human embryonic kidney (HEK)-293T cell line was purchased from the Institute of Basic Medical Sciences, Chinese Academy of Medical Sciences (Beijing, China) and maintained in Dulbecco's modified Eagle's medium (Hyclone; Cytiva) containing 10\% fetal calf serum (Gibco; Thermo Fisher Scientific, Inc.). Both cell lines were kept in a humidified atmosphere of $5 \% \mathrm{CO}_{2}$ at $37^{\circ} \mathrm{C}$.

Cell transfection. Empty pcDNA3.1 vector, ACSS2 overexpression plasmid (pcDNA3.1/ACSS2), miR-15b mimics/inhibitors and mimic/inhibitor negative controls (NC) were obtained from RiboBio Co., Ltd.. At $24 \mathrm{~h}$ before the transient transfections, an appropriate number of human aortic VSMCs were seeded into six-well plates with $2 \mathrm{ml}$ of SMCM and grown to $60-70 \%$ confluency at $37^{\circ} \mathrm{C}$ in an atmosphere containing $5 \% \mathrm{CO}_{2}$. Then, the aortic VSMCs were divided into six groups, and miR-15b-5p mimic NC (5'-UCACAACCUCCUAGAAAGAGUAGA-3'; double stranded; $100 \mathrm{nM}$ ), miR-15b-5p mimic (5'-UAGCAG CACAUCAUGGUUUACA-3'; double stranded; $100 \mathrm{nM}$ ), miR-15b-5p inhibitor NC (5'-UCUACUCUUUCUAGGAGG UUGUGA-3'; single stranded; $200 \mathrm{nM})$, miR-15b-5p inhibitor (5'-AUCGUCGUGUAGUACCAAAUGU-3'; single stranded; $200 \mathrm{nM}$ ), pcDNA3.1 plasmid (4 $\mu \mathrm{g} / \mathrm{well})$, pcDNA3.1/ACSS2 plasmid (4 $\mu \mathrm{g} /$ well), miR-15b-5p mimics+pcDNA3.1 plasmid, or miR-15b-5p mimics+pcDNA3.1/ACSS2 plasmid were added into the plates. The transient transfections of these mimics or inhibitors were performed using the Exfect Transfection reagent (Vazyme Biotech Co.) according to the manufacturer's instructions.

Cell proliferation assay. At $60-70 \%$ confluence, human aortic VSMCs $\left(2 \times 10^{3} /\right.$ well $)$ were plated into 96 -well plates with $200 \mu \mathrm{l}$ of SMCM and underwent the different transfection treatments as aforementioned. Cell viability was determined at $0,24,48$ and $72 \mathrm{~h}$ post-transfection using the Cell Counting Kit-8 (CCK-8; Beyotime Institute of Biotechnology). The absorbance of each well was measured using a microplate reader (MK3; Thermo Fisher Scientific, Inc.) at $450 \mathrm{~nm}$.

Cell apoptosis assay. At 60-70\% confluence, human aortic VSMCs $\left(2 \times 10^{3} /\right.$ well $)$ were plated into six-well plates with $2 \mathrm{ml}$ of SMCM and subjected to various transfection treatments as aforementioned. At $48 \mathrm{~h}$ after transfection, human aortic VSMCs were digested with $0.25 \%$ trypsin for 3-5 min, centrifuged at $1,000 \times \mathrm{g}$ for $5 \mathrm{~min}$ and washed with precooled phosphate-buffered saline twice. Cell pellets were resuspended with $1 \mathrm{X}$ binding buffer at a density of $10^{6}$ cells $/ \mathrm{ml}$. A total of $10^{5}$ cells were then stained with $5 \mu \mathrm{l}$ Annexin $\mathrm{V}$ and $5 \mu \mathrm{l}$ propidium iodide for $15 \mathrm{~min}$ at room temperature in the dark (MultiSciences Biotech Co., Ltd.). Cell apoptosis was analyzed by flow cytometry using CytExpert software (version 2.0; Beckman Coulter, Inc.).

$R T-q P C R$. At $48 \mathrm{~h}$ after transfection, human aortic VSMCs were treated with TRIzol ${ }^{\circledR}$ reagent (Tiangen Biotech Co., Ltd.) to isolate the total RNA. For amplification of ACSS2, total RNA was reverse transcribed to cDNA using the FastQuant cDNA synthesis kit (Tiangen Biotech Co., Ltd.), and then qPCR was performed using SuperReal PreMix (Tiangen Biotech Co., Ltd.). For amplification of miR-15b-5p, first strand cDNA synthesis was conducted with the miRNA 1st-Strand 


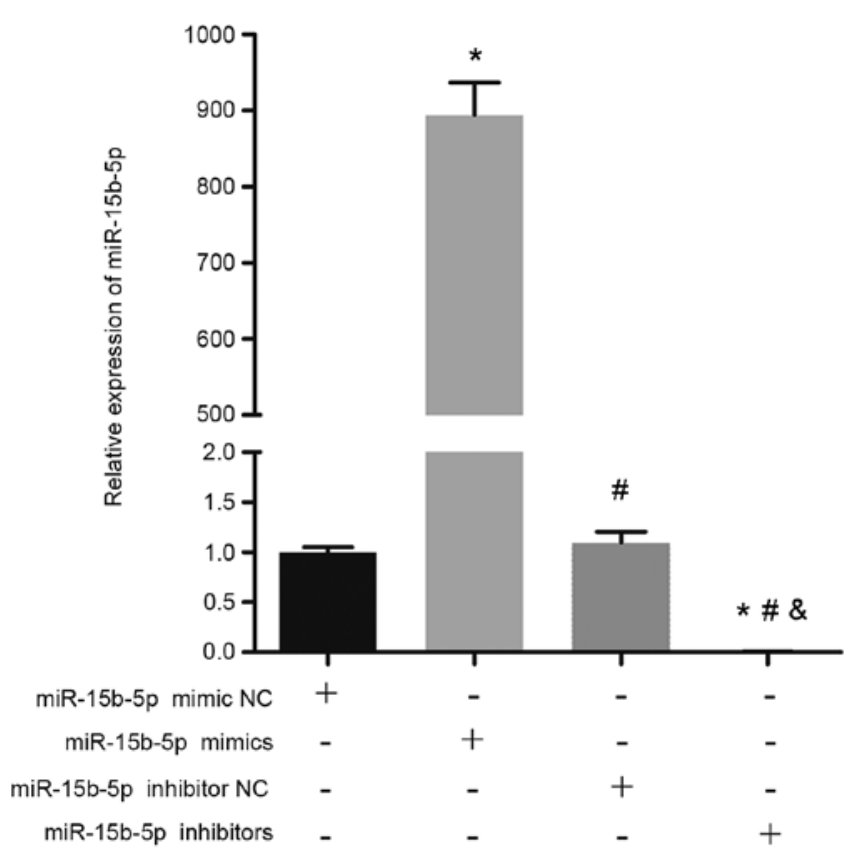

Figure 1. Expression levels of miR-15b-5p in human aortic vascular smooth muscle cells after different transfections, as determined by reverse transcription quantitative-PCR. Results are presented as the average+standard deviation of three independent experiments. ${ }^{*} \mathrm{P}<0.05$ vs. mimic NC; ${ }^{~} \mathrm{P}<0.05$ vs. miR-15b-5p mimics; ${ }^{\circledR} \mathrm{P}<0.05$ vs. inhibitor NC. miR, microRNA; NC, negative control.

cDNA Synthesis kit (by stem-loop; Vazyme Biotech Co. Ltd.), and qPCR was run according to the instructions of the miRNA Universal SYBR qPCR Master Mix (Vazyme Biotech Co., Ltd.). Primers were synthesized by the Beijing Dingguo Changsheng Biotechnology Co., Ltd., and were as follows: ACSS2, CGTGATGGGGCTTCCTGAG (forward) and GTT GTACCGAAGGAATGGGC (reverse); miR-15b-5p, CGC GTAGCAGCACATCATGG (forward) and GTCGTATCC AGTGCAGGGTCCGAGGTATTCGCACTGGATACGACT GTAAA (reverse); $\beta$-actin, ACACTGTGCCCATCTACG (forward) and TGTCACGCACGATTTCC (reverse); and U6, CTCGCTTCGGCAGCACA (forward) and AACGCTTCA CGAATTTGCGT (reverse). $\beta$-actin and U6 served as an endogenous reference for mRNAs and miRNAs, respectively. The PCR procedure was: Denaturation at $95^{\circ} \mathrm{C}$ for $15 \mathrm{~min}$, followed by 40 cycles of $95^{\circ} \mathrm{C}$ for $10 \mathrm{sec}, 60^{\circ} \mathrm{C}$ for $20 \mathrm{sec}$ and $72^{\circ} \mathrm{C}$ for $30 \mathrm{sec}$. The relative expression levels of mRNAs and miRNAs were determined using the $2^{-\Delta \Delta C q}$ method (17).

Western blot analysis. At $48 \mathrm{~h}$ after transfection, human aortic VSMCs were treated with the RIPA lysis buffer (Solarbio Science \& Technology Co., Ltd.) to extract the total proteins. The concentration of total proteins was determined using the BCA Protein Assay kit (Beyotime Institute of Biotechnology). The protein samples $(30 \mu \mathrm{g})$ were separated by $10 \%$ sodium dodecyl sulfonate-polyacrylamide gel electrophoresis and then electrophoretically transferred to polyvinylidene fluoride membranes (EMD Millipore). After being blocked with $5 \%$ skim milk for $1 \mathrm{~h}$ at room temperature, the membranes were incubated with primary antibodies against ACSS2 (1:2,000; cat. no. 16087-1-AP; ProteinTech Group, Inc.), prostaglandin-endoperoxide synthase 2 (PTGS2; 1:2,000; cat. no. 27308-1-AP; ProteinTech Group, Inc.) and GAPDH
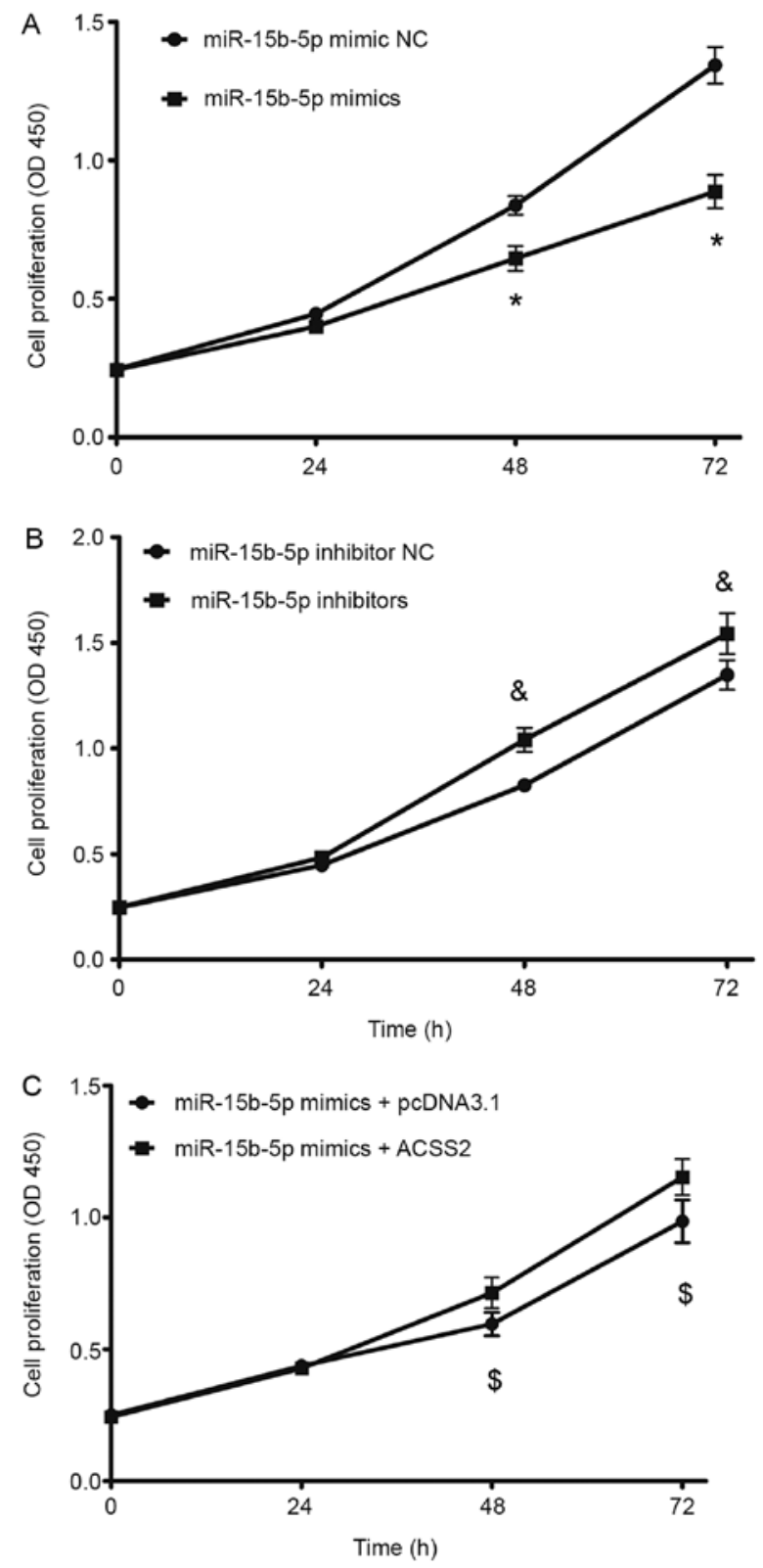

Figure 2. Proliferation of human aortic VSMCs after different transfections, as determined by CCK-8 assay. (A) miR-15b-5p mimics and NC groups. (B) miR-15b-5p inhibitors and NC groups. (C) Rescue experiments by simultaneous overexpression of ACSS2 in aortic VSMCs transfected with miR-15b-5p mimics. The OD was detected at $0,24,48$ and $72 \mathrm{~h}$ after transfection. Results are presented as the average \pm standard deviation of four independent experiments. ${ }^{*} \mathrm{P}<0.05$ vs. mimic $\mathrm{NC}$; ${ }^{\&} \mathrm{P}<0.05$ vs. inhibitor $\mathrm{NC} ;{ }^{~} \mathrm{P}<0.05$ vs. miR-15b-5p mimics+pcDNA3.1 empty vector. VSMCs, vascular smooth muscle cells; miR, microRNA; NC, negative control; ACSS2, acyl-CoA synthetase short chain family member 2; OD, optical density.

(1:1,000; cat. no. ab181602; Abcam) overnight at $4^{\circ} \mathrm{C}$. The membranes were then incubated with the horseradish peroxidase-conjugated secondary antibody at room temperature for 1 h (1:5,000; cat. no. E030120; EarthOx Life Sciences). Protein bands were visualized using an enhanced chemiluminescence kit (Beyotime Institute of Biotechnology) and quantified using ImageJ software (version 1.46; National Institutes of Health). GAPDH was used as an internal control. Protein expression levels were calculated as relative to the mimic NC group, which was set as 1.0. 

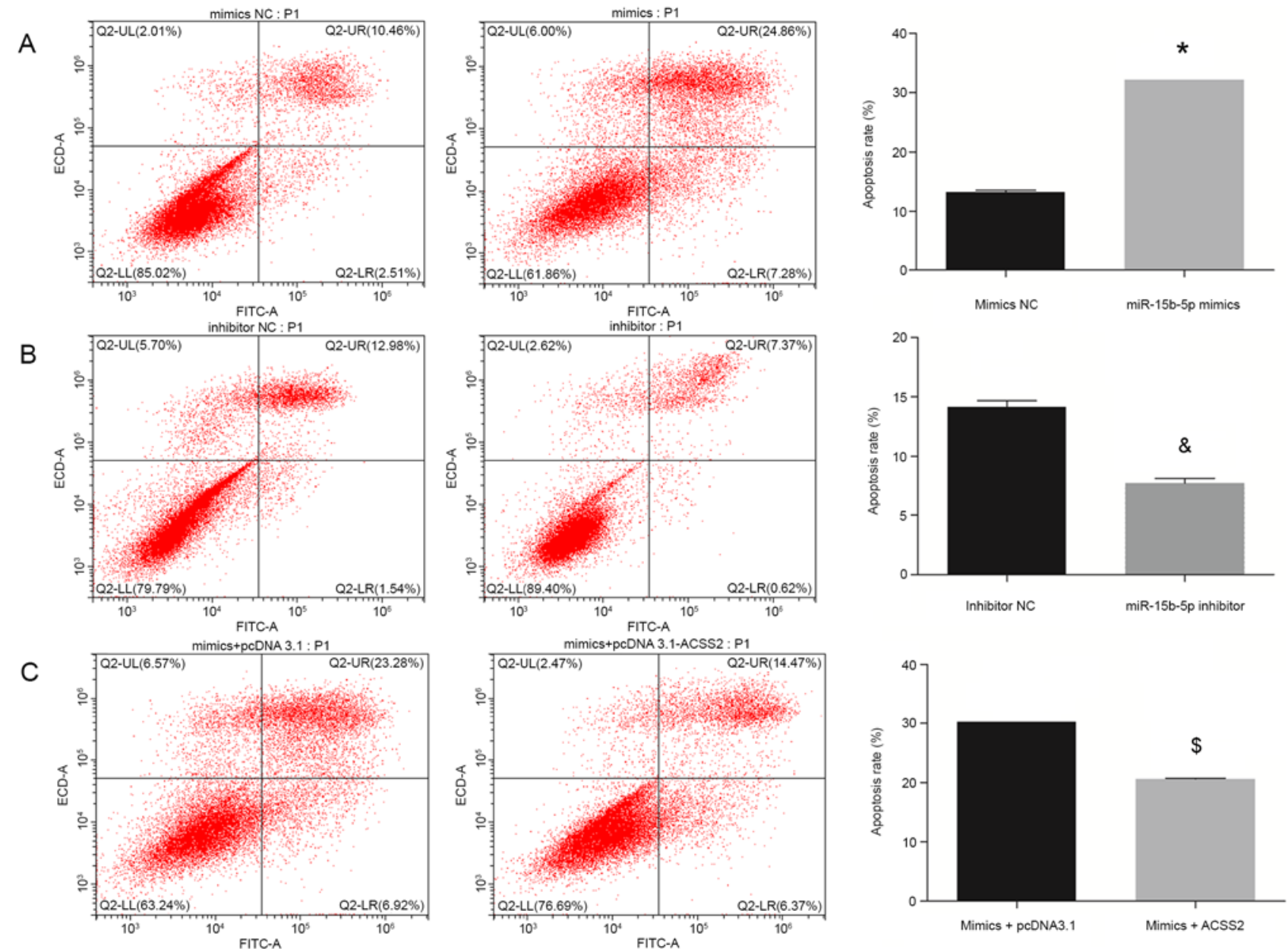

Figure 3. Apoptosis of human aortic VSMCs at $48 \mathrm{~h}$ after different transfections, as determined by flow cytometry assay. (A) miR-15b-5p mimics and NC groups. (B) miR-15b-5p inhibitors and NC groups. (C) Rescue experiments by simultaneous overexpression of ACSS2 in aortic VSMCs transfected with miR-15b-5p mimics. Representative flow cytometry plots and quantifications are shown. Results are presented as the average+standard deviation of two independent experiments. ${ }^{\text {P }}<0.05$ vs. mimic $\mathrm{NC} ;{ }^{\&} \mathrm{P}<0.05$ vs. inhibitor $\mathrm{NC} ;{ }^{\$} \mathrm{P}<0.05$ vs. miR-15b-5p mimics+pcDNA3.1 empty vector. VSMCs, vascular smooth muscle cells; miR, microRNA; NC, negative control; ACSS2, acyl-CoA synthetase short chain family member 2.

Luciferase reporter assay. TargetScan software (version 7.0; targetscan.org/) (18) was used to identify the miR-15b-5p binding site in the 3'-UTR of ACSS2. The wild-type (WT) and mutant (MUT) 3'-UTR sequences of ACSS2 at the miR-15b binding site were chemically synthesized by General Biosystems, Inc., and inserted into the XhoI/NotI site of psiCHECK-2 luciferase vector (Promega Corporation) to construct the recombinant plasmids psiCHECK2-ACSS2-WT and psiCHECK2-ACSS2-MUT, respectively. miR-15b-5p mimics or miR-15b-5p mimic NC were co-transfected with psiCHECK2-ACSS2-WT and psiCHECK2-ACSS2-MUT into HEK-293T cells using the Exfect Transfection reagent (Vazyme Biotech Co., Ltd.). After 48 h, firefly and Renilla luciferase activities were measured on an EnSpire reader (PerkinElmer, Inc.) using the Dual Luciferase Assay System (Dalian Meilun Biology Technology Co., Ltd.).

Statistical analysis. All experiments were performed at least two independent times. Data were expressed as mean \pm standard deviation. Differences between two groups were analyzed by Student's t-test. Differences among more than two groups were tested using one-way analysis of variance followed by post hoc Tukey's (equal variances) or Dunnett's
T3 (unequal variances) test. Statistical analysis was conducted using SPSS 18.0 (SPSS, Inc.) and graphs were generated in GraphPad Prism 5.0 (GraphPad Software, Inc.). P $<0.05$ was considered to indicate a statistically significant difference.

\section{Results}

Construction of in vitro transfection models. To explore the potential roles of miR-15-5p, in vitro overexpression and knockdown models were first established by transfecting the human aortic VSMCs with miR-15b-5p mimics and miR-15b-5p inhibitors, respectively. As shown in Fig. 1, the expression levels of miR-15b-5p were significantly increased in the miR-15b-5p mimic group compared with those in the mimic NC group. By contrast, the expression levels of miR-15b-5p were significantly lower in the miR-15b-5p inhibitor group compared with those in the inhibitor NC group (Fig. 1). These findings indicated that overexpression and knockdown of miR-15b-5p were successfully established in human aortic VSMCs.

miR-15b-5p has an antiproliferative role in aortic VSMCs. CCK-8 assay was used to evaluate the impact of miR-15b-5p 
A

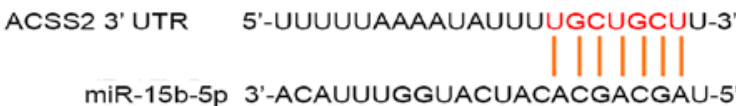

ACSS2 3' UTR MUT 5'-UUUUUAAAAUAUUUACGACGAU-3

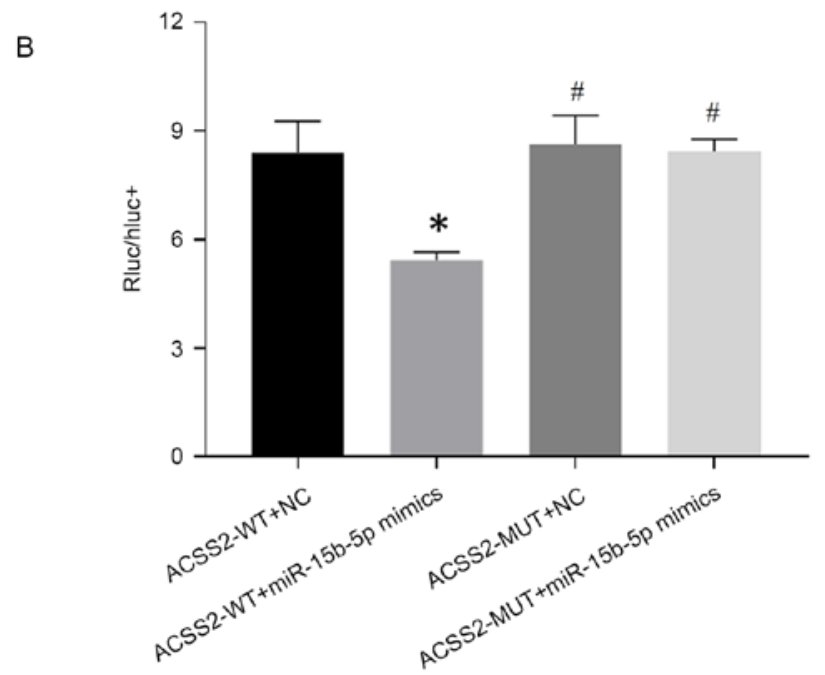

Figure 4. ACSS2 is a direct target of miR-15b-5p. (A) Schematic of the predicted binding site between miR-15b-5p and the 3'-UTR of ACSS2 (B) Luciferase reporter assays were conducted to confirm the binding ability between miR-15b-5p and ACSS2. WT or MUT constructs of the 3'-UTR of ACSS2 were co-transfected with miR-15b-5p mimics or NC in HEK-293T cells. The firefly luciferase activity was normalized against Renilla luciferase activity. Results are presented as the average+standard deviation of four independent experiments. ${ }^{\text {P }}<0.05$ vs. mimic NC; ${ }^{\#} \mathrm{P}<0.05$ vs. miR-15b-5p mimics. ACSS2, acyl-CoA synthetase short chain family member 2; miR, microRNA; UTR, untranslated region; WT, wild-type; MUT; mutant; NC, negative control.

on the proliferation of human aortic VSMCs. The results demonstrated that the absorbance value of aortic VSMCs was significantly lower at 48 and $72 \mathrm{~h}$ after transfection with miR-15b-5p mimics compared with the mimic NC group, but no statistical difference was observed at $24 \mathrm{~h}$ (Fig. 2A). By contrast, transfection with miR-15b-5p inhibitors resulted in a significant increase in cell proliferation compared with the inhibitor NC group, showing a higher absorbance value at 48 and $72 \mathrm{~h}$ after transfection (Fig. 2B). These findings suggested that miR-15b-5p may have an antiproliferative role in aortic VSMCs. The time point of $48 \mathrm{~h}$ post-transfection was selected for subsequent analyses.

miR-15b-5p exerts proapoptotic activity in aortic VSMCs. Annexin V-FITC/PI staining and flow cytometry analysis were used to investigate the effects of miR-15b-5p on the apoptosis of aortic VSMCs. As shown in Fig. 3A, the apoptosis rate of aortic VSMCs transfected with miR-15b-5p mimics was found to be increased by $\sim 2.5$-fold compared with that in cells transfected with the mimic NC. By contrast, transfection with the miR-15b-5p inhibitors was associated with a 1.8 -fold decrease in the proportion of apoptotic cells in aortic VSMCs when compared with the inhibitor NC group treatment (Fig. 3B). These findings indicated that miR-15b-5p may exert proapoptotic activity in aortic VSMCs.

miR-15b-5p regulates the expression of ACSS2 in aortic VSMCs. Bioinformatic analysis predicted that the 3'-UTR region of ACSS2 had the binding site for miR-15b-5p (Fig. 4A). To explore the regulatory relationship of ACSS2 and miR-15b-5p, a dual-luciferase reporter assay in HEK-293T cells was conducted. As expected, the luciferase activity of the ACSS2-WT vector was significantly inhibited by miR-15b-5p mimics compared with the mimic NC, while no significant change was detected in the luciferase activity of the ACSS2-MUT reporter between the miR-15b-5p mimics and mimic NC groups (Fig. 4B), implying the ACSS2 may be a direct target of miR-15b-5p.

To further confirm their interaction, the expression levels of ACSS2 were measured in aortic VSMCs following different transfections. In line with the dual-luciferase reporter assay, both the mRNA and protein expression levels of ACSS2 in aortic VSMCs were found to be significantly reduced after transfection with miR-15b-5p mimics, but significantly increased after transfection with miR-15b-5p inhibitors (Fig. 5B-D).

miR-15b-5p regulates the proliferation and apoptosis of aortic VSMCs via targeting ACSS2. To further explore whether the proapoptotic and antiproliferative effects of miR-15b-5p was achieved through ACSS2, rescue experiments were performed. Firstly, pcDNA3.1/ACSS2 was confirmed to have an effective transfection efficiency (Fig. 5A). Then, miR-15b-5p-mediated suppression of ACSS2 was demonstrated to be rescued by simultaneous overexpression of ACSS2 in aortic VSMCs (Fig. 5B-D). Functional assays demonstrated that overexpression of ACSS2 reversed the effects of miR-15b-5p mimics on aortic VSMCs, resulting in an increase in cell proliferation (Fig. 2C), and a decrease in cell apoptosis (Fig. 3C), compared with the miR-15b-5p+empty pcDNA3.1 vector group. These findings suggested that miR-15b-5p-induced apoptosis may be rescued by ACSS 2 .

Furthermore, our previous bioinformatics prediction analysis revealed that miR-15b-5p may function in the development of AAA by regulating ACSS2 and then affecting the lipid biosynthetic process (16). PTGS2 (also known as COX-2) is a key regulatory enzyme in lipid metabolism, which was also upregulated in AAA (16). Thus, it can be hypothesized that PTGS2 may be a downstream target of the miR-15b-5p/ACSS2 interaction. To validate this hypothesis, the protein expression levels of PTGS2 were detected in all six transfection groups. In accordance with its expression in AAA, PTGS2 was demonstrated to be highly expressed in the miR-15b-5p mimic group, but significantly reduced in the miR-15b-5p inhibitor group compared with their corresponding controls. Additionally, overexpression of ACSS2 could partially block the production of PTGS2 protein (Fig. 5C and E).

\section{Discussion}

The present study, for the first time, demonstrated that overexpression of miR-15b-5p promoted the apoptosis and inhibited the proliferation of human aortic VSMCs by downregulating the expression of ACSS2 and subsequently upregulating PTGS2. Of note, silencing of miR-15b-5p obtained the opposite results. The present findings were in line with the pathological feature of aortic VSMCs loss in AAA $(6,19,20)$ and the observed expression levels of miR-15b-5p (upregulated), 
A
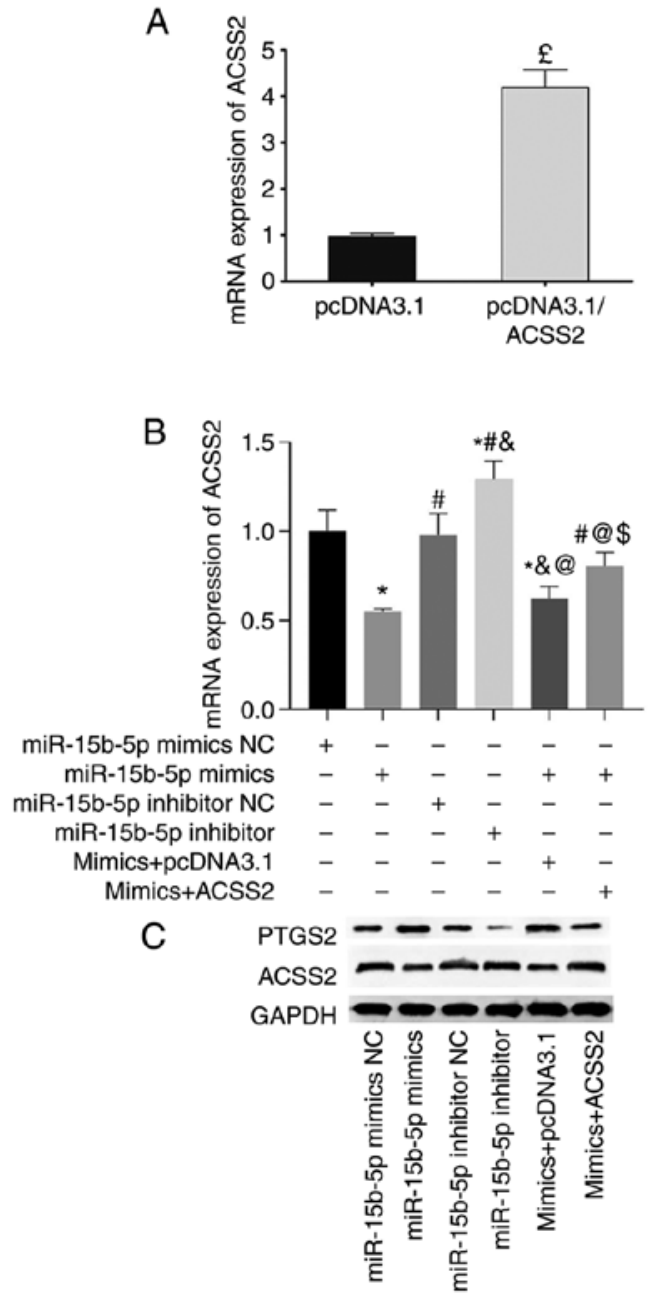

D

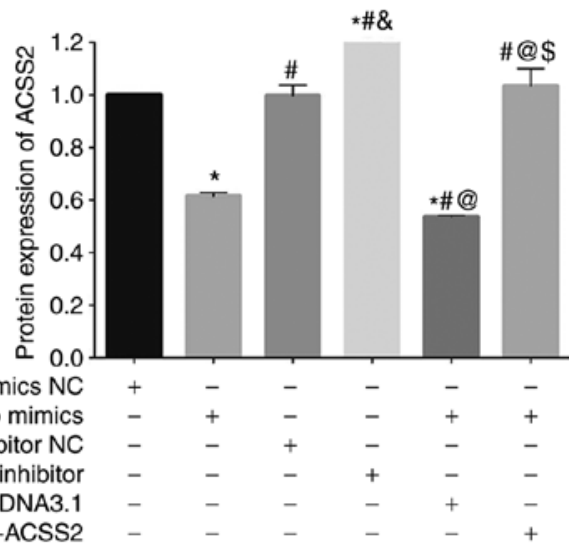

$\mathrm{E}$

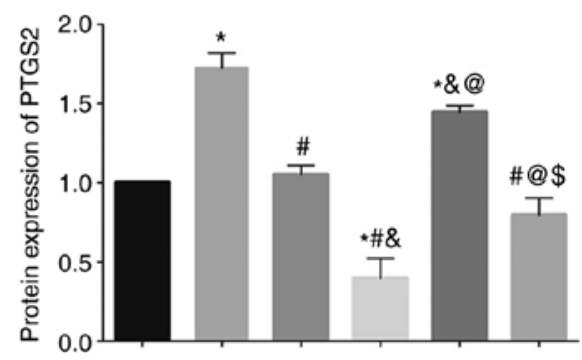

$\begin{array}{rcccccc}\text { miR-15b-5p mimics NC } & + & - & - & - & - & - \\ \text { miR-15b-5p mimics } & - & + & - & - & + & + \\ \text { miR-15b-5p inhibitor NC } & - & - & + & - & - & - \\ \text { miR-15b-5p inhibitor } & - & - & - & + & - & - \\ \text { Mimics+pcDNA3.1 } & - & - & - & - & + & - \\ \text { Mimics+ACSS2 } & - & - & - & - & - & +\end{array}$

Figure 5. ACSS2 and PTGS2 expression levels are regulated by miR-15b-5p. (A) RT-qPCR was performed to confirm the successful overexpression of ACSS2. (B) RT-qPCR was performed to measure the mRNA expression levels of ACSS2 after miR-15b-5p transfection treatments. (C) Representative images from western blot analysis showing the protein expression levels of ACSS2 and PTGS2 in the different transfection groups. (D) Quantitative results of ACSS2 protein. (E) Quantitative results of PTGS2 protein. Results are presented as the average+standard deviation of three independent experiments for mRNAs and two indepen-

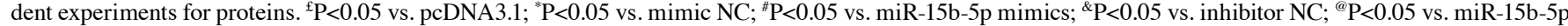
inhibitors; ${ }^{\mathrm{S}} \mathrm{P}<0.05$ vs. miR-15b-5p mimics+pcDNA3.1 empty vector. ACSS2, acyl-CoA synthetase short chain family member 2; PTGS2, prostaglandin-endoperoxide synthase 2; miR, microRNA; RT-qPCR, reverse transcription-quantitative PCR; NC, negative control.

ACSS2 (downregulated) and PTGS2 (upregulated) in AAA samples (16). Therefore, the hsa-miR-15b-5p/ACSS2 interaction axis may be an important contributor to the development of AAA by regulating the proliferation and apoptosis of human aortic VSMCs and may represent attractive therapeutic molecules for AAA.

Although there were no studies to explore the role of miR-15b-5p in AAA, its function in cancer may indirectly explain the importance of miR-15b-5p for the development and progression of AAA. Dong et al (21) reported that miR-15b-5p was upregulated in liver cancer tissues and cell lines, and that miR-15b-5p levels were associated with high tumor-node-metastasis stage, tumor capsular infiltration and shorter overall survival rates. Overexpression of miR-15b-5p promoted liver cancer cell proliferation and invasion (21). Zhao et al (22) identified that miR-15b-5p was highly expressed in gastric cancer cell lines, tissues, and plasma samples in comparison with normal controls. Ectopic expression of miR-15b-5p in gastric cancer cells accelerated cell proliferation, migration, invasion and epithelial-mesenchymal transition (22). Wu et al (23) observed that knockdown of miR-15b-5p inhibited growth and invasiveness, and induced apoptosis of breast cancer cells and significantly restrained tumor growth in a xenograft model in mice. These findings indicated that miR-15b-5p may be an oncogenic driver. Because depletion of aortic VSMCs is a common pathological feature in AAA, the present study speculated that miR-15b-5p may also be upregulated in AAA samples similar to cancer, and then it may promote the apoptosis of aortic VSMCs. The present study confirmed this hypothesis, demonstrating that miR-15b mimics significantly reduced the viability and facilitated the apoptosis of human VSMCs, and that opposite effects were observed following miR-15b inhibitor transfection. Further confirmation of this hypothesis was reported in a recent study by Sun et al (24).

The known downstream target genes of miR-15b-5p include axin 2 (21), progestin and adipo $Q$ receptor family member 3 (22), heparanase 2 (23), and reversion inducing cysteine rich protein with kazal motifs (25) in cancer cells, and the insulin like growth factor 1 receptor (24) in human VSMCs. The present study, for the first time, demonstrated that ACSS2 may also be a direct 
target of miR-15b-5p using the dual-luciferase reporter system. In addition, ACSS2 overexpression could reverse the proapoptotic and antiproliferative effects of miR-15b-5p on aortic VSMCs. In consideration of the roles of miR-15b-5p in AAA and cancer, it can be speculated that ACSS2 may serve as a tumor suppressor gene. This hypothesis has been supported by several previous studies: Hur et al (26) and Bae et al (27) identified that downregulation of ACSS2 is an independent factor to predict poor survival of patients with gastric cancer and colorectal carcinoma, respectively. Knockdown of ACSS2 was shown to increase the invasion and migration ability of hepatocellular carcinoma cells by promoting the epithelial-mesenchymal transition (28). However, the tumor suppressor mechanisms of ACSS2 were rarely reported because more scholars believe that ACSS2 may be oncogenic (29-31) by catalyzing the production of acetyl-CoA from acetate, CoA and ATP and then promoting fatty acid synthesis as cell membrane component, single molecular or energy source in tumor cells (32-34). The present study demonstrated that ACSS2 may exert tumor suppressor roles by inhibiting the expression of PTGS2. This negative regulatory relationship between ACSS2 and PTGS2 may be associated with the ability of ACSS2 to drive acetylation of the target genes and then reduce their activity (28). Furthermore, considerable evidence revealed that the expression of PTGS2 was increased in an AAA animal model and patients (35); the use of a PTGS2 inhibitor (36-38) or genetic deficiency of PTGS2 (39) significantly reduced the incidence, severity, aortic rupture and mortality in AAA model mice. PTGS2 may be involved in the development of AAA due to its ability to convert arachidonic acid into prostaglandins. Prostaglandin E2 was found to inhibit DNA synthesis, proliferation and death of aortic VSMCs derived from aneurysmal aorta by activation of a serial of inflammatory pathways (40). In line with these studies, the current findings also revealed that the expression of PTGS2 was increased in VSMCs with higher apoptosis rates.

There were several limitations in the present study. Firstly, the present study provided preliminary evidence to show that the miR-15b-5p/ACSS2/PTGS2 axis may be a potential target for the treatment of AAA by changing the apoptosis and proliferation of aortic VSMCs. In vivo animal models need to be established in order to confirm the therapeutic effects of anti-miR-15b-5p in AAA prior to clinical application. Secondly, it will be valuable to collect aortic samples from patients with AAA from our hospital to validate the expression of miR-15b-5p/ACSS2/PTGS2 and their association with clinical features (such as aneurysm diameter and rupture incidence). Thirdly, the downstream mechanisms of ACSS2 need to be further explored in the future, by overexpression and knockdown of ACSS2 and by miR-15b-5p and PTGS2 (or other genes) rescue experiments.

In conclusion, the present study suggested that miR-15b-5p may promote the apoptosis and inhibit the proliferation of aortic VSMCs via targeting the ACSS2/PTGS2 axis. Targeted regulation of the miR-15b-5p/ACSS2/PTGS2 axis may serve as a potential approach for the treatment of AAA, a disease characterized pathologically by loss of VSMCs.

\section{Acknowledgements}

Not applicable.

\section{Funding}

The present study was supported by the Special Disease of Vasculitis in Pudong New Area (grant no. PWZzb2017-07), the Outstanding Clinical Discipline Project of Shanghai Pudong (grant no. PWYgy-2018-08) and the Program for Medical Key Department of Shanghai (grant no. ZK2019A10).

\section{Availability of data and materials}

All data generated or analyzed during this study are included in this published article.

\section{Authors' contributions}

SG, JT and ZQ contributed to the conception and design of this study. SG performed the experiments and the statistical analyses. JM and YP were involved in the interpretation of the data. SG and JT drafted the manuscript. ZQ edited and revised the manuscript. SG and ZQ confirm the authenticity of all the raw data. All authors read and approved the final manuscript.

\section{Ethics approval and consent to participate}

Not applicable.

\section{Patient consent for publication}

Not applicable.

\section{Competing interests}

The authors declare that they have no competing interests.

\section{References}

1. Kent KC: Clinical practice. Abdominal aortic aneurysms. N Engl J Med 371: 2101-2108, 2014.

2. Dereziński TL, Fórmankiewicz B, Migdalski A, Brazis P, Jakubowski G, Woda $€$ and Jawień A: The prevalence of abdominal aortic aneurysms in the rural/urban population in central Poland-Gniewkowo aortic study. Kardiol Pol 75: 705-710, 2017.

3. Stuntz M: Modeling the burden of abdominal aortic aneurysm in the USA in 2013. Cardiology 135: 127-131, 2016.

4. Kühnl A, Erk A, Trenner M, Salvermoser M, Schmid V and Eckstein $\mathrm{HH}$ : Incidence, treatment and mortality in patients with abdominal aortic aneurysms. Dtsch Arztebl Int 114: 391-398, 2017.

5. Lederle FA, Kyriakides TC, Stroupe KT, Freischlag JA, Padberg FT Jr, Matsumura JS, Huo Z and Johnson GR; OVER Veterans Affairs Cooperative Study Group: Open versus endovascular repair of abdominal aortic aneurysm. N Engl J Med 380: 2126-2135, 2019.

6. Thompson RW, Liao S and Curci JA: Vascular smooth muscle cell apoptosis in abdominal aortic aneurysms. Coron Artery Dis 8: 623-631, 1997.

7. Liang B, Che J, Zhao H, Zhang Z and Shi G: MiR-195 promotes abdominal aortic aneurysm media remodeling by targeting Smad3. Cardiovasc Ther 35: e12286, 2017.

8. Zhao F, Chen $\mathrm{T}$ and Jiang N: CDR1as/miR-7/CKAP4 axis contributes to the pathogenesis of abdominal aortic aneurysm by regulating the proliferation and apoptosis of primary vascular smooth muscle cells. Exp Ther Med 19: 3760-3766, 2020.

9. Cao X, Cai Z, Liu J, Zhao Y, Wang X, Li X and Xia H: miRNA-504 inhibits p53-dependent vascular smooth muscle cell apoptosis and may prevent aneurysm formation. Mol Med Rep 16: 2570-2578, 2017.

10. Zhang Y, Liu Z, Zhou M and Liu C: MicroRNA-129-5p inhibits vascular smooth muscle cell proliferation by targeting Wnt5a. Exp Ther Med 12: 2651-2656, 2016. 
11. Zhao L, Ouyang Y, Bai Y, Gong J and Liao H: miR-155-5p inhibits the viability of vascular smooth muscle cell via targeting FOS and ZIC3 to promote aneurysm formation. Eur J Pharmacol 853 : 145-152, 2019.

12. Yue J, Zhu T, Yang J, Si Y, Xu X, Fang Y and Fu W: CircCBFB-mediated miR-28-5p facilitates abdominal aortic aneurysm via LYPD3 and GRIA4. Life Sci 253: 117533, 2020.

13. Maegdefessel L, Azuma J, Toh R, Deng A, Merk DR, Raiesdana A, Leeper NJ, Raaz U, Schoelmerich AM, McConnell MV, et al: MicroRNA-21 blocks abdominal aortic aneurysm development and nicotine-augmented expansion. Sci Transl Med 4: 122ra22, 2012.

14. Peng J, He X, Zhang L and Liu P: MicroRNA-26a protects vascular smooth muscle cells against $\mathrm{H} 2 \mathrm{O} 2$-induced injury through activation of the PTEN/AKT/mTOR pathway. Int J Mol Med 42: 1367-1378, 2018.

15. Shen G, Sun Q, Yao Y, Li S, Liu G, Yuan C, Li H, Xu Y and Wang H: Role of ADAM9 and miR-126 in the development of abdominal aortic aneurysm. Atherosclerosis 297: 47-54, 2020.

16. Gan S, Pan Y and Mao J: miR-30a-GNG2 and miR-15b-ACSS2 interaction pairs may be potentially crucial for development of abdominal aortic aneurysm by influencing inflammation. DNA Cell Biol 38: 1540-1556,2019.

17. Livak KJ and Schmittgen TD: Analysis of relative gene expression data using real-time quantitative PCR and the 2(-Delta Delta C(T)) method. Methods 25: 402-408, 2001.

18. Agarwal V, Bell GW, Nam JW and Bartel DP: Predicting effective microRNA target sites in mammalian mRNAs. Elife 4: e05005, 2015.

19. Zhang Z, Zou G, Chen X, Lu W, Liu J, Zhai S and Qiao G: Knockdown of lncRNA PVT1 inhibits vascular smooth muscle cell apoptosis and extracellular matrix disruption in a murine abdominal aortic aneurysm model. Mol Cells 42: 218-227, 2019.

20. Xue M, Li G, Li D, Wang Z, Mi L, Da J and Jin X: Up-regulated MCPIP1 in abdominal aortic aneurysm is associated with vascular smooth muscle cell apoptosis and MMPs production. Biosci Rep 39: BSR20191252, 2019.

21. Dong Y, Zhang N, Zhao S, Chen X, Li F and Tao X: miR-221-3p and miR-15b-5p promote cell proliferation and invasion by targeting Axin2 in liver cancer. Oncol Lett 18: 6491-6500, 2019.

22. Zhao C, Li Y, Chen G, Wang F, ShenZ and Zhou R: Overexpression of miR-15b-5p promotes gastric cancer metastasis by regulating PAQR3. Oncol Rep 38: 352-358, 2017.

23. Wu B, Liu G, Jin Y, Yang T, Zhang D, Ding L, Zhou F, Pan Y and Wei Y: miR-15b-5p promotes growth and metastasis in breast cancer by targeting HPSE2. Front Oncol 10: 108, 2020.

24. Sun Y, Gao Y, Song T, Yu C, Nie Z and Wang X: MicroRNA-15b participates in the development of peripheral arterial disease by modulating the growth of vascular smooth muscle cells. Exp Ther Med 18: 77-84, 2019.

25. Chen R, Sheng L, Zhang HJ, Ji M and Qian WQ: miR-15b-5p facilitates the tumorigenicity by targeting RECK and predicts tumour recurrence in prostate cancer. J Cell Mol Med 22: 1855-1863, 2018.

26. Hur H, Kim YB, Ham IH and Lee D: Loss of ACSS2 expression predicts poor prognosis in patients with gastric cancer. J Surg Oncol 112: 585-591, 2015

27. Bae JM, Kim JH, Oh HJ, Park HE, Lee TH, Cho NY and Kang GH: Downregulation of acetyl-CoA synthetase 2 is a metabolic hallmark of tumor progression and aggressiveness in colorectal carcinoma. Mod Pathol 30: 267-277, 2017.
28. Sun L, Kong Y, Cao M, Zhou H, Li H, Cui Y, Fang F, Zhang W, $\mathrm{Li}$ J, Zhu X, et al: Decreased expression of acetyl-CoA synthase 2 promotes metastasis and predicts poor prognosis in hepatocellular carcinoma. Cancer Sci 108: 1338-1346, 2017.

29. Yao L, Guo X and Gui Y: Acetyl-CoA synthetase 2 promotes cell migration and invasion of renal cell carcinoma by upregulating lysosomal-associated membrane protein 1 expression. Cell Physiol Biochem 45: 984-992, 2018.

30. Zhang S, He J, Jia Z, Yan Z and Yang J: Acetyl-CoA synthetase 2 enhances tumorigenesis and is indicative of a poor prognosis for patients with renal cell carcinoma. Urol Oncol 36: 243.e9-243. e20, 2018

31. Mi L, Zhou Y, Wu D, Tao Q, Wang X, Zhu H, Gao X, Wang J, Ling R, Deng J, et al: ACSS2/AMPK/PCNA pathway-driven proliferation and chemoresistance of esophageal squamous carcinoma cells under nutrient stress. Mol Med Rep 20: 5286-5296, 2019.

32. Huang Z,Zhang M,Plec AA, Estill SJ, Cai L, Repa JJ, McKnight SL and Tu BP: ACSS2 promotes systemic fat storage and utilization through selective regulation of genes involved in lipid metabolism. Proc Natl Acad Sci USA 115: E9499-E9506, 2018.

33. Lee MY, Yeon A, Shahid M, Cho E, Sairam V, Figlin R, Kim KH and Kim J: Reprogrammed lipid metabolism in bladder cancer with cisplatin resistance. Oncotarget 9: 13231-13243, 2018.

34. Xu H, Luo J, Ma G, Zhang X, Yao D, Li M and Loor JJ: Acyl-CoA synthetase short-chain family member 2 (ACSS2) is regulated by SREBP-1 and plays a role in fatty acid synthesis in caprine mammary epithelial cells. J Cell Physiol 233: 1005-1016, 2018.

35. Chapple KS, Parry DJ, McKenzie S, MacLennan KA, Jones P and Scott DJ: Cyclooxygenase-2 expression and its association with increased angiogenesis in human abdominal aortic aneurysms. Ann Vasc Surg 21: 61-66, 2007.

36. Ghoshal S and Loftin CD: Cyclooxygenase-2 inhibition attenuates abdominal aortic aneurysm progression in hyperlipidemic mice. PLoS One 7: e44369, 2012

37. King VL, Trivedi DB, Gitlin JM and Loftin CD: Selective cyclooxygenase-2 inhibition with celecoxib decreases angiotensin II-induced abdominal aortic aneurysm formation in mice. Arterioscler Thromb Vasc Biol 26: 1137-1143, 2006.

38. Mukherjee K, Gitlin JM and Loftin CD: Effectiveness of cyclooxygenase- 2 inhibition in limiting abdominal aortic aneurysm progression in mice correlates with a differentiated smooth muscle cell phenotype. J Cardiovasc Pharmacol 60: 520-529, 2012.

39. Gitlin JM, Trivedi DB, Langenbach R and Loftin CD: Genetic deficiency of cyclooxygenase- 2 attenuates abdominal aortic aneurysm formation in mice. Cardiovasc Res 73: 227-236, 2007.

40. Walton LJ, Franklin IJ, Bayston T, Brown LC, Greenhalgh RM, Taylor GW and Powell JT: Inhibition of prostaglandin E2 synthesis in abdominal aortic aneurysms: Implications for smooth muscle cell viability, inflammatory processes, and the expansion of abdominal aortic aneurysms. Circulation 100: 48-54, 1999.

(i) (5) This work is licensed under a Creative Commons Attribution-NonCommercial-NoDerivatives 4.0 International (CC BY-NC-ND 4.0) License. 